\section{Conhecimento dos agentes comunitários de saúde sobre a tuberculose, suas medidas de controle e tratamento diretamente observado}

\author{
Community health workers' knowledge on \\ tuberculosis, control measures, and \\ directly observed therapy
}

\section{Conocimiento de agentes comunitarios de salud sobre la tuberculosis, medidas de control y tratamiento directamente observado}

\begin{abstract}
This study assessed community health workers' (CHW) knowledge on tuberculosis (TB), TB control measures, and directly observed therapy (DOT) in Belo Horizonte, Minas Gerais State, Brazil. This was a cross-sectional study that ap plied a questionnaire in a random and representative sample of $489 \mathrm{CHW}$. The overall proportion of correct answers was $74.6 \%$. The mean proportions of correct answers in the domains for TB, TB control measures, and DOT were $81 \%, 84.1 \%$, and $59.4 \%$, respectively. The study showed knowledge gaps in the identification of patients with pulmonary $T B$, the target public for DOT, and adequate technique for treatment supervision. Better knowledge was associated with time working as a $\mathrm{CHW}(\mathrm{OR}=2.3)$ and follow-up of cases in the 12 months prior to the interview $(O R=1.7)$. The study provided new scientific data on level of knowledge among CHW concerning DOT and can support strategies to improve TB control activities.
\end{abstract}

Community Health Workers; Health Knowledge, Attitudes, Practice; Tuberculosis
Gustavo Silva Souto Rocha 1,2

Marina Guimaraes Lima 3

Joyce Laura Moreira 2

Kennedy Crepalde Ribeiro 1

Maria das Graças Braga Ceccato 3

Wânia da Silva Carvalho ${ }^{3}$

Micheline Rosa Silveira ${ }^{3}$

\section{Resumo}

Trata-se de estudo de corte transversal, realizado mediante a aplicação de questionário em uma amostra aleatória e representativa de 489 agentes comunitários de saúde (ACS) a respeito da tuberculose (TB), suas medidas de controle e o tratamento diretamente observado (TDO), em Belo Horizonte, Minas Gerais, Brasil. A proporção média de itens acertados foi de 74,6\%. A proporção média de acertos no domínio TB foi $81 \%$; no domínio medidas de controle, $84,1 \%$; e sobre TDO, 59,4\%. Verificou-se uma lacuna no conhecimento sobre a identificação de pacientes com TB pulmonar, o público-alvo do TDO, e a técnica adequada para supervisão do tratamento. Foi observada associação entre maior conhecimento e tempo de atuação igual ou superior a três anos $(R C=2,3)$ e acompanhamento de casos nos 12 meses anteriores à entrevista $(R C=1,7)$. Este trabalho apresentou informações inéditas na literatura científica sobre o nível de conhecimento dos ACS sobre TDO, e poderá subsidiar estratégias destinadas ao aperfeiçoamento das atividades de controle da tuberculose.

Agentes Comunitários de Saúde; Conhecimentos, Atitudes e Prática em Saúde; Tuberculose 


\section{Introdução}

A tuberculose (TB) apresenta forte componente social e releva-se como problema de saúde em escala mundial, permanecendo como segunda causa entre as mortes por doenças infecciosas e sendo responsável por 8,6 milhões de casos com 1,3 milhão de mortes associadas à doença 1 . O Brasil está incluído entre os 22 países com a maior carga da doença, responsáveis por cerca de $80 \%$ do total de casos existentes mundialmente e que recebe particular atenção para o controle de TB desde 2000 2. No país, foram notificados em 2013, 71.123 casos novos da doença. A taxa de incidência foi de 35,4/100 mil habitantes e a mortalidade igual a 2,3/100 mil habitantes. Por sua vez, no mesmo ano, o Estado de Minas Gerais apresentou taxa de incidência igual a 17,9/100 mil habitantes, registrando tendência de queda, em concordância com os dados nacionais. Ainda no mesmo ano, a capital do estado, Belo Horizonte, apresentou taxa de incidência de 26,4/ 100 mil habitantes e as taxas de mortalidade registradas por estado e capital foram de 1,4 e 1,5 por 100 mil habitantes/ano, respectivamente. Ainda em 2013, o país registrou 70,6\% de cura e 10,5\% de abandono, o Estado de Minas Gerais registrou $64,2 \%$ de cura e $9,1 \%$ de abandono, e sua capital, Belo Horizonte, alcançou $65,2 \%$ de cura e $14 \%$ de abandono 3 .

Em 1993, devido à conjuntura epidemiológica da TB no mundo, a Organização Mundial da Saúde (OMS) recomendou a implementação do Direct Observed Treatment Short-Course (DOTS) como estratégia de atuação dos países membros no controle da doença. A partir de 1996, ano em que foi divulgado o Plano Emergencial para o Controle da Tuberculose, o Ministério da Saúde passou a recomendar a realização do DOTS, no entanto, a estratégia foi implantada efetivamente em 1999 pelo Programa Nacional de Controle da Tuberculose (PNCT) 4,5. A estratégia DOTS significa mais que o tratamento diretamente observado (TDO), incluindo a adesão política das autoridades, a implantação de rede laboratorial de baciloscopia, a garantia de medicamentos e o adequado sistema de informação ${ }^{6}$. As perspectivas relacionadas ao DOTS são a redução da taxa de abandono, a atenuação do surgimento de resistência entre os bacilos e o efetivo controle da TB 4

Para o profissional de saúde, recomenda-se além da visualização da ingestão do medicamento, a criação de vínculo e responsabilidade entre paciente e serviço de saúde. A observação da tomada de medicamentos deve ser realizada diariamente, nos dias úteis, tanto no serviço de saúde quanto no domicílio. No entanto, para fins de notificação ao Sistema de Informação de Agravos de Notificação (SINAN), convencionouse que ao final do tratamento o paciente deverá ter no mínimo 24 tomadas observadas na fase de ataque e 48 na fase de manutenção 7 .

A execução da vigilância da tomada de medicamentos influencia diretamente a atuação dos profissionais envolvidos com a Estratégia Saúde da Família (ESF) e, em especial, o agente comunitário de saúde (ACS), que é o profissional designado para aumentar o vínculo de pacientes e comunidade com a Unidade Básica de Saúde (UBS). No país, atualmente, existem 284 mil ACS em exercício registrados no Cadastro Nacional de Estabelecimentos de Saúde (CNES; http://cnes. datasus.gov.br/, acessado em 26/Jun/2014). Esses profissionais atuam, de maneira individual ou coletiva, em atividades de prevenção de doenças e promoção de saúde. Para o exercício profissional é pré-requisito ser residente na área da comunidade onde trabalha, ter concluído o Ensino Fundamental e ter finalizado o curso introdutório de formação inicial e continuada. Em razão do controle da TB, espera-se que o ACS realize, além da supervisão do tratamento, outras ações como identificar pacientes sintomáticos respiratórios nos domicílios e comunidade; encaminhar casos suspeitos às UBS; confirmar se os contatos foram avaliados e examinados; encaminhar os contatos à UBS para consulta, diagnóstico e tratamento quando necessários; preencher a ficha de acompanhamento do TDO; verificar a situação vacinal de crianças contactantes de casos de TB; e realizar busca ativa de faltosos e daqueles que abandonarem o tratamento 8 .

Entre habilidades e conhecimentos necessários à atuação como ACS, está a capacidade de articulação dos saberes técnicos e populares. Portanto, o conhecimento a respeito da TB e das formas de atuação profissional para controle da doença pode proporcionar a efetividade na execução das tarefas dos ACS, dentre elas a conscientização dos pacientes a respeito da doença $\mathrm{e}$ a melhoria nas taxas de detecção e abandono 9 . O conhecimento remete ao conceito de compreensão. Para atingi-lo é necessário que o indivíduo estabeleça conexões entre elementos informacionais, armazene, processe, analise, relacione e avalie segundo critérios de relevância 10. Atualmente, existem poucos estudos nacionais e internacionais que abordam ou tentam mensurar o conhecimento de profissionais de saúde em relação à TB 11,12. As pesquisas que tratam do conhecimento sobre TB tendo como cerne o ACS são ainda mais escassas. Entrevistas realizadas com ACS de Vitória (Espírito Santo) resultaram na associação do maior conhecimento desses profissionais com um maior tempo de atuação 
profissional, entretanto não foi avaliado o nível de conhecimento sobre TDO 13.

Da identificação de lacunas no conhecimento dos profissionais de saúde pode surgir a contribuição para o planejamento de ações que busquem aperfeiçoar a atuação no controle da doença e a reorientação da gestão clínica dos casos nos serviços de saúde. Diante desse contexto, o presente estudo pretende avaliar o nível de conhecimento dos ACS a respeito da TB, suas medidas de controle e o TDO, e verificar fatores associados aos mesmos no Município de Belo Horizonte.

\section{Métodos}

\section{Delineamento e local da pesquisa}

O estudo possui delineamento transversal. Os dados foram coletados entre julho e dezembro de 2013, no Município de Belo Horizonte, que apresenta uma população aproximada de 2,37 milhões de habitantes e densidade demográfica igual a 7.167 habitantes $/ \mathrm{km}^{2}$, segundo o Censo Demográfico de 2010 (Instituto Brasileiro de Geografia e Estatística. Censo demográfico 2010. http://censo2010.ibge.gov.br/, acessado em 07/ Out/2015). A rede de saúde municipal conta com 147 centros de saúde (CS), distribuídos em nove Distritos Sanitários (DS). A população total de ACS, registrados no CNES, em Belo Horizonte, no período do estudo era igual a 2.720 .

\section{Amostragem do estudo}

O cálculo do tamanho mínimo da amostra foi realizado com base no total de 2.720 ACS cadastrados no CNES. Foram considerados: prevalência a priori de $50 \%$ devido ao desconhecimento da proporção do evento, nível de significância de 5\%, intervalo de 95\% de confiança (IC95\%), efeito de desenho igual a 1 e perda estimada de $30 \%$, totalizando uma amostra mínima de 438 ACS. Para considerar na composição da amostra a proporção de ACS em cada um dos DS de Belo Horizonte e o perfil dos CS segundo a classificação do abono de estímulo à fixação profissional estabelecida pela Secretaria Municipal de Saúde de Belo Horizonte, optou-se por ampliar o número de ACS a serem entrevistados para 679. Essa opção foi realizada devido à heterogeneidade dos centros de saúde no município com relação ao perfil de saúde da população e às condições de trabalho aos quais os ACS são submetidos, que refletem na classificação do abono de estímulo à fixação profissional das unidades. Todas as unidades de saúde de Belo Horizonte estão categorizadas, de acordo com a legislação vigen- te, em valores crescentes de abonos de fixação, A, B, C ou D; de acordo com sua localização, acessibilidade, dificuldade de lotação de pessoal e prioridade administrativa. Segundo esse critério, as unidades classificadas com a letra D são consideradas as mais críticas pela administração municipal, portanto, seus trabalhadores recebem um maior abono salarial em relação às outras unidades $14,15,16$.

O processo de definição da composição da amostra foi realizado em duas etapas. Inicialmente, definiu-se o número de ACS em cada DS na amostra considerando-se a proporção de ACS segundo o DS na população total. Posteriormente, designou-se o número de ACS a serem entrevistados segundo categoria de abono de fixação, levando-se em consideração o percentual de CS em cada categoria de abono e o número de ACS a serem entrevistados em cada DS. Em seguida, definiu-se o número de CS a serem incluídos na pesquisa, considerando a proporção de ACS por DS e por abono salarial, resultando em um total de 37 CS. Foram gerados números aleatórios no software OpenEpi OpenEpi (Dean AG, Sullivan KM, Soe MM. OpenEpi: Open Source Epidemiologic Statistics for Public Health; http://www. OpenEpi.com) para os centros de saúde de cada categoria de abono e DS. Realizou-se o sorteio dos mesmos para a inclusão na amostra. A quantidade de ACS que estavam registrados no CNES pelos CS sorteados incluídos na amostra totalizou 679. Portanto, apesar do tamanho mínimo da amostra ter sido definido estatisticamente em 438 ACS, procurou-se entrevistar os 679 ACS das 37 unidades sorteadas para que a amostra fosse representativa das características da população total de ACS e daquela distribuída por CS de Belo Horizonte.

Ao término da coleta de dados, dos 679 ACS, 617 estavam efetivamente em exercício, 497 compareceram para entrevista nos dias e locais previamente agendados e 489 concordaram em participar da pesquisa $(\mathrm{N}=489)$. Os oito ACS que se recusaram a participar não informaram o motivo da recusa.

A distribuição da amostra segundo DS apresentou 81 (16,6\%) ACS entrevistados no Barreiro, $30(6,1 \%)$ no Centro-sul, $42(8,6 \%)$ no Leste, 73 $(14,9 \%)$ no Nordeste, $48(9,8 \%)$ no Noroeste, 49 (10,0\%) no Norte, 52 (10,6\%) no Oeste, 51 (10,4\%) no Pampulha e $63(12,9 \%)$ no DS Venda Nova. O número de CS sorteados foi maior nos DS Barreiro e Nordeste com cinco CS em cada um, e menor no DS Venda Nova com três CS. Os demais DS apresentaram quatro CS sorteados. Observou-se sete $(18,9 \%)$ CS incluídos com abono fixação A, nove $(24,3 \%)$ com abono B, 12 (32,4\%) com C e nove $(24,3 \%)$ com D. 


\section{Coleta de dados}

Os dados foram apurados com base nas respostas dadas pelos ACS a um questionário semiestruturado, autoaplicável e pré-testado. A avaliação foi constituída de perguntas fechadas e utilizou-se como referências bibliográficas para a elaboração das questões o Manual de Recomendações para Controle da Tuberculose no Brasil 7, o livro Tratamento Diretamente Observado (TDO) da Tuberculose na Atenção Básica: Protocolo de Enfermagem 17 e a cartilha Tuberculose: Orientações para Agentes Comunitários de Saúde 18.

A coleta dos dados ocorreu entre os meses de julho e dezembro de 2013, após a realização de projeto piloto com subsequente incorporação das adequações identificadas como necessárias, tanto nos instrumentos quanto no fluxo das ações a serem desenvolvidas.

Foram considerados elegíveis para a pesquisa todos os ACS que aceitaram participar e assinaram o Termo de Consentimento Livre e Esclarecido (TCLE).

\section{Variáveis}

Para fins de medida de resultado, o nível de conhecimento dos ACS foi mensurado segundo três domínios: TB, medidas de controle e TDO. Cada domínio foi avaliado baseando-se em um número variável de questões, sendo 12 sobre TB, oito sobre medidas de controle e dez sobre TDO. Considerou-se que a resposta selecionada em cada questão era um indicador do nível de conhecimento dos ACS. Em cada item, os participantes poderiam responder entre sim, não ou não sei.

As variáveis explicativas investigadas foram agrupadas em três categorias: características sociodemográficas, características do serviço de saúde e características de atuação em TB. Dentre as características do serviço de saúde coletadas encontra-se o Índice de Vulnerabilidade à Saúde (IVS), indicador adotado em Belo Horizonte para classificar o risco à saúde das áreas e microáreas vinculadas aos setores censitários localizados no município 19 .

\section{Análise dos dados}

Os dados obtidos foram organizados em uma planilha no software SPSS 19.0 (IBM Corp., Armonk, Estados Unidos). Para a medida de resultado as respostas foram consideradas dicotômicas (sim/não). Quando os ACS não respondiam ou selecionavam a resposta não sei, a alternativa foi considerada incorreta. A interpretação dos resultados foi realizada por dois revisores e nos casos de discordância um terceiro revisor foi consultado. Todos os dados digitados foram conferidos por um segundo pesquisador e realizouse a dupla digitação de $10 \%$ dos questionários aplicados para avaliação de concordância. Em seguida, o número de acertos de cada ACS em cada domínio do conhecimento e o número total de acertos no teste foi calculado. Baseando-se no total de acertos categorizou-se o conhecimento do ACS em abaixo ou acima da mediana.

Estimou-se em uma análise univariada a magnitude da associação entre as variáveis explicativas e o nível de conhecimento acima da mediana por meio do teste do qui-quadrado, adotando-se o nível de significância de 5\%. As variáveis com valor de p igual ou menor que 0,25 no teste de Wald e aquelas de relevância epidemiológica, na análise univariada, foram selecionadas manualmente para iniciar o modelo multivariado com o procedimento passo a passo com seleção para trás. O nível de significância exigido para inclusão no modelo final foi de 0,05 , para melhor averiguar potenciais fatores de confusão. Permaneceram no modelo final apenas as variáveis que demonstraram associação independente significativa com o evento de interesse. Foi calculada a razão de chances (RC) com IC95\% para cada variável estudada. Utilizou-se o teste da razão de verossimilhança para comparar os modelos. A adequação final dos modelos foi avaliada pelo teste de Hosmer-Lemeshow. As análises estatísticas foram feitas usando-se o software SPSS, versão 19.0 .

\section{Considerações éticas}

O projeto de pesquisa foi aprovado pelo Comitê de Ética da Universidade Federal de Minas Gerais e pelo Comitê de Ética em Pesquisa da Secretaria Municipal de Saúde de Belo Horizonte (CAAE: 14046913.5.0000.5149).

\section{Resultados}

Com relação às características sociodemográficas dos ACS, foi observado que a maioria dos respondentes é do sexo feminino (92,6\%). A idade média dos ACS foi igual a 42,1 $( \pm 9,6)$ anos, variando entre 23 e 74 anos, e 39,5\% estavam na faixa etária entre 30 e 39 anos. Em relação à escolaridade, 88,1\% relataram ter concluído o Ensino Médio. O tempo médio de atuação profissional foi igual a $8,3( \pm 4,1)$ anos e $24,5 \%$ dos ACS trabalhavam por 12 anos ou mais (Tabela 1).

Considerando-se as características dos serviços de saúde, mais especificamente o IVS do município, 14,3\% dos respondentes trabalhavam em microárea de risco baixo, $39,1 \%$ em risco 
Características sociodemográficas dos agentes comunitários de saúde. Belo Horizonte, Minas Gerais, Brasil, 2013.

\begin{tabular}{|c|c|c|}
\hline Variáveis & $n(N=489 *)$ & $\%$ \\
\hline \multicolumn{3}{|l|}{ Gênero } \\
\hline Feminino & 453 & 92,6 \\
\hline Masculino & 33 & 6,8 \\
\hline \multicolumn{3}{|l|}{ Faixa etária (anos) } \\
\hline $18-29$ & 43 & 8,8 \\
\hline $30-39$ & 193 & 39,5 \\
\hline $40-49$ & 140 & 28,6 \\
\hline 50 ou mais & 107 & 21,9 \\
\hline \multicolumn{3}{|l|}{ Nível de escolaridade } \\
\hline Ensino Fundamental & 21 & 4,3 \\
\hline Ensino Médio incompleto & 36 & 7,4 \\
\hline Ensino Médio completo & 364 & 74,4 \\
\hline Ensino Superior incompleto & 44 & 9,0 \\
\hline Ensino Superior completo & 21 & 4,3 \\
\hline Pós-graduação & 2 & 0,4 \\
\hline \multicolumn{3}{|l|}{ Estado civil } \\
\hline Solteiro & 133 & 27,2 \\
\hline Casado & 249 & 50,9 \\
\hline Divorciado & 51 & 10,4 \\
\hline União estável & 40 & 8,2 \\
\hline Viúvo & 14 & 2,9 \\
\hline \multicolumn{3}{|l|}{ Tempo de atuação (anos) ** } \\
\hline $0-3$ & 37 & 7,6 \\
\hline $3-6$ & 122 & 24,9 \\
\hline $6-9$ & 62 & 12,7 \\
\hline $9-12$ & 64 & 13,1 \\
\hline 12 ou mais & 120 & 24,5 \\
\hline
\end{tabular}

* A soma das frequências não é igual a 100\%, devido à exclusão dos não respondentes;

** $17,2 \%$ de não respondentes.

médio, $25,6 \%$ em risco elevado e $16,6 \%$ em risco muito elevado. Dentre os ACS, $89 \%$ acompanhavam mais de 150 famílias, com média de 250,2 famílias por profissional, e 59,1\% participavam de ESF completa. Para 21,3\%, o profissional em falta na equipe foi o ACS, representando percentual maior que aqueles $9,8 \%$ que citaram o profissional médico como ausente. A responsabilidade pela supervisão das ações profissionais no controle da TB foi atribuída ao enfermeiro por $45,2 \%$ dos ACS e pelo enfermeiro em conjunto com outras categorias profissionais por $33,1 \%$ dos entrevistados (Tabela 2).

Como demonstrado na Tabela 3, dentre os ACS, $69,7 \%$ relataram acompanhamento prévio de casos de TB dentro da microárea em que atuavam e 44,8\% dos respondentes acompanharam um ou mais casos de TB durante os 12 meses an- teriores à entrevista. Quanto à realização do TDO, $52,4 \%$ dos ACS executaram-no ao menos uma vez durante o tempo de atuação profissional e $42,9 \%$ preencheram a ficha de acompanhamento da tomada diária da medicação em alguma ocasião. $\mathrm{O}$ abandono de casos entre os pacientes acompanhados foi relatado por $23,5 \%$ dos ACS e 69,5\% afirmaram participação em capacitação específica sobre TB. Dentre os respondentes, $50,9 \%$ afirmaram realizar ações educativas e informativas sobre a doença junto à comunidade.

Nos 12 itens referentes ao nível de conhecimento do domínio TB, foi observada uma média de acerto de 9,7 ou $81 \%$ das questões. No domínio medidas de controle, a média de acerto correspondeu a $84,1 \%$ das questões e no domínio TDO foi de $59,4 \%$ (Tabela 4). A média de itens acertados entre os respondentes foi igual a 22,4 
Tabela 2

Características dos serviços de saúde relatadas pelos agentes comunitários de saúde. Belo Horizonte, Minas Gerais, Brasil, 2013.

\begin{tabular}{|c|c|c|}
\hline Variáveis & $n(N=489 *)$ & $\%$ \\
\hline \multicolumn{3}{|l|}{ Classificação IVS da microárea } \\
\hline Risco baixo & 70 & 14,3 \\
\hline Risco médio & 191 & 39,1 \\
\hline Risco elevado & 125 & 25,6 \\
\hline Risco muito elevado & 81 & 16,5 \\
\hline Mais de um IVS na microárea & 17 & 3,5 \\
\hline \multicolumn{3}{|l|}{ Número de famílias acompanhadas } \\
\hline Até 150 & 32 & 6,5 \\
\hline Mais de 150 & 435 & 89,0 \\
\hline \multicolumn{3}{|l|}{ Vínculo com eSF } \\
\hline Sim & 482 & 98,6 \\
\hline Não & 5 & 1,0 \\
\hline \multicolumn{3}{|l|}{ eSF completa } \\
\hline Sim & 289 & 59,1 \\
\hline Não & 196 & 40,1 \\
\hline \multicolumn{3}{|l|}{ Profissionais em falta na eSF } \\
\hline ACS & 104 & 21,3 \\
\hline Auxiliar de Enfermagem & 4 & 0,8 \\
\hline Enfermeiro & 4 & 0,8 \\
\hline Médico & 48 & 9,8 \\
\hline Mais de uma categoria profissional & 29 & 5,9 \\
\hline Sem falta de profissional & 289 & 59,1 \\
\hline \multicolumn{3}{|c|}{$\begin{array}{l}\text { Profissional responsável pela supervisão das ações no } \\
\text { controle da TB }\end{array}$} \\
\hline Enfermeiro & 221 & 45,2 \\
\hline Médico & 4 & 0,8 \\
\hline Farmacêutico & 2 & 0,4 \\
\hline Enfermeiro e outras categorias & 162 & 33,1 \\
\hline Mais de duas categorias profissionais & 67 & 13,7 \\
\hline \multicolumn{3}{|l|}{ Avaliação da supervisão } \\
\hline Insatisfatória & 13 & 2,7 \\
\hline Regular & 50 & 10,2 \\
\hline Satisfatória & 261 & 53,4 \\
\hline Excelente & 123 & 25,1 \\
\hline
\end{tabular}

ACS: agentes comunitários de saúde; eSF: equipe de Saúde da Família; IVS: índice de vulnerabilidade à saúde; TB: tuberculose.

* A soma das frequências não é igual a 100\%, devido à exclusão dos não respondentes.

$( \pm 3,0)$ ou $74,6 \%$ do total de questões. Por fim, com base no total de acertos de cada indivíduo no questionário, constituído por trinta questões, os ACS foram categorizados pelo conhecimento abaixo ou acima da mediana, localizada no corte de acerto de 23 questões. Foi observado que 263 ou 53,8\% dos ACS entre os entrevistados apresentaram conhecimento acima da mediana.
Mediante análise univariada demonstrada na Tabela 5, observou-se que as características sociodemográficas que apresentaram associação significativa com o nível de conhecimento dos ACS acima da mediana foram a faixa etária de até 39 anos, nível de escolaridade correspondente a Ensino Médio completo ou Superior e tempo de atuação profissional igual ou acima de três anos. 
Tabela 3

Características dos agentes comunitários de saúde na atuação para o controle da tuberculose. Belo Horizonte, Minas Gerais, Brasil, 2013.

\begin{tabular}{|c|c|c|}
\hline Variáveis & $n(N=489 *)$ & $\%$ \\
\hline \multicolumn{3}{|l|}{ TB intradomiciliar } \\
\hline Sim & 61 & 12,5 \\
\hline Não & 426 & 87,1 \\
\hline \multicolumn{3}{|c|}{ Caso de TB na microárea } \\
\hline Sim & 341 & 69,7 \\
\hline Não & 147 & 30,1 \\
\hline \multicolumn{3}{|c|}{ Casos de TB assistidos nos últimos 12 meses } \\
\hline Nenhum & 258 & 52,8 \\
\hline 1 ou mais & 219 & 44,8 \\
\hline \multicolumn{3}{|l|}{ Realizou TDO } \\
\hline Sim & 256 & 52,4 \\
\hline Não & 225 & 46,0 \\
\hline \multicolumn{3}{|c|}{ Preencheu ficha de acompanhamento } \\
\hline Sim & 210 & 42,9 \\
\hline Não & 278 & 56,9 \\
\hline \multicolumn{3}{|c|}{ Abandono de casos entre pacientes } \\
\hline Sim & 115 & 23,5 \\
\hline Não & 371 & 75,9 \\
\hline \multicolumn{3}{|l|}{ Capacitação sobre TB } \\
\hline Sim & 340 & 69,5 \\
\hline Não & 146 & 29,9 \\
\hline \multicolumn{3}{|c|}{ Quantidade de capacitações } \\
\hline 1 & 169 & 34,6 \\
\hline 2 & 95 & 19,4 \\
\hline 3 ou mais & 21 & 4,3 \\
\hline \multicolumn{3}{|l|}{ Última capacitação } \\
\hline Menos de 6 meses & 47 & 9,6 \\
\hline 6 meses a 1 ano & 75 & 15,3 \\
\hline $1-2$ anos & 139 & 28,4 \\
\hline Mais de 2 anos & 91 & 18,6 \\
\hline \multicolumn{3}{|c|}{ Realiza ações educativas sobre TB } \\
\hline Sim & 249 & 50,9 \\
\hline Não & 227 & 46,4 \\
\hline
\end{tabular}

TB: tuberculose; TDO: tratamento diretamente observado.

* A soma das frequências não é igual a 100\%, devido à exclusão dos não respondentes;

As características dos serviços de saúde não apresentaram associação estatisticamente significativa com o nível de conhecimento na análise univariada. Contudo, quanto à atuação para controle da TB, o acompanhamento de algum caso da doença durante o tempo de atuação profissional, o seguimento de algum caso de TB nos últimos 12 meses, a experiência prática no TDO, a experiência prática no preenchimento da ficha de acompanhamento e a participação em capa- citações específicas sobre TB apresentaram associação significativa com o nível de conhecimento.

Mediante a análise multivariada entre o nível de conhecimento acima da mediana e as características dos ACS que demonstraram significância estatística, foram observadas associações entre o tempo de atuação do ACS igual a três anos ou mais $(\mathrm{RC}=2,3)$ e o acompanhamento de um ou mais casos de casos de TB pelos ACS nos 12 meses anteriores à entrevista $(\mathrm{RC}=1,7)$. 
Tabela 4

Frequência de acertos dos agentes comunitários de saúde $(N=489)$ por item do questionário de avaliação.

\section{Afirmativa}

Resposta correta
Frequência de acertos [n (\%)]

Domínio: TB (12 questões)

Trata-se de uma doença infecto-contagiosa

É causada por vírus

É transmitida por picada de mosquito

É transmitida sexualmente

É transmitida por meio da tosse, espirro ou fala

Transmite-se principalmente por pacientes com TB dos pulmões e da laringe

Transmite-se por pacientes com TB ocular

Transmite-se por pacientes acometidos por TB cutânea

É comum pacientes com TB apresentarem febre

O principal sintoma da TB pulmonar é a tosse

Pacientes com TB podem apresentar suor excessivo durante a noite

Pacientes com TB podem apresentar perda de peso

Domínio: Medidas de controle (8 questões)

Uma das atividades realizadas pelo ACS no controle da TB é identificar todos os contatos do

caso e orientá-los quanto à realização de exames

É atividade do ACS verificar a situação vacinal de crianças em contato com casos de TB

O ACS deve orientar quanto à necessidade do paciente utilizar máscara cirúrgica em domicílio

O ACS pode contribuir com orientações para os pacientes e familiares sobre o risco de

transmissão da doença em casos sem tratamento ou com menos de 15 dias de uso do

medicamento

Uma das atividades realizadas pelo ACS no acompanhamento de casos de TB consiste em

realizar busca ativa de faltosos e daqueles que abandonam o tratamento

É responsabilidade do ACS levar os pacientes para consultas médicas realizadas fora da área de abrangência do Centro de Saúde

É responsabilidade do ACS realizar orientações ao paciente preferencialmente em ambiente fechado para maior humanização do cuidado

É atividade do ACS orientar o paciente que ele deve finalizar o tratamento sem interrupções para atingir a cura da doença

Domínio: TDO (10 questões)

Destina-se somente aos pacientes com TB pulmonar

Destina-se principalmente aos pacientes com TB latente

Destina-se aos pacientes com TB e AIDS

O TDO pode ser executado por qualquer profissional de saúde

De acordo com as normas do PNCT do Ministério da Saúde, o TDO pode ser realizado por familiares

Deve ser realizado somente na residência do paciente

Inclui também o preenchimento da ficha de acompanhamento da tomada diária da medicação

O ACS deve entregar ao familiar a ficha de acompanhamento da tomada diária da medicação

para que ele preencha

A frequência mínima recomendada para a realização do TDO é três vezes na semana, durante

todo o tratamento

O TDO inclui visitas obrigatórias aos finais de semana
$9,7(81,0)$

$482(98,6)$

$185(37,8)$

$471(96,3)$

$436(89,2)$

$483(98,8)$

$431(88,1)$

$237(48,5)$

$275(56,2)$

$408(83,4)$

$464(94,9)$

$411(84,1)$

$470(96,1)$

$6,7(84,1)$

$479(98,0)$

$475(97,1)$

$165(33,7)$

$472(96,5)$

$489(100,0)$

$468(95,7)$

$268(54,8)$

$474(96,9)$

$5,9(59,4)$

$231(47,2)$

$164(33,5)$

$213(43,6)$

$331(67,7)$

$133(27,2)$

$367(75,1)$

$465(95,1)$

$291(59,5)$

$304(62,2)$

$406(83,0)$

ACS: agentes comunitários de saúde; PNCT: Programa Nacional de Controle da Tuberculose; TB: tuberculose; TDO: tratamento diretamente observado. 
Análises univariada e multivariada das características dos agentes comunitários de saúde associadas ao nível de conhecimento acima da mediana *. Belo Horizonte, Minas Gerais, Brasil, 2013.

\begin{tabular}{|c|c|c|c|c|c|c|}
\hline \multirow[t]{2}{*}{ Variáveis ** } & \multicolumn{4}{|c|}{ Análise univariada } & \multicolumn{2}{|c|}{ Análise multivariada } \\
\hline & $\mathbf{N}$ & $\begin{array}{l}\text { Conhecimento acima } \\
\text { da mediana }\end{array}$ & RC (IC95\%) & Valor de p & RC (IC95\%) & Valor de $p$ \\
\hline Faixa etária (anos) & & & & 0,006 & & \\
\hline Até 39 & 236 & $142(60,2)$ & 1,00 & & & \\
\hline 40 ou mais & 247 & $117(47,4)$ & $0,60(0,42 ; 0,85)$ & & & \\
\hline Nível de escolaridade & & & & $<0,001$ & & \\
\hline $\begin{array}{l}\text { Ensino Fundamental ou Ensino Médio } \\
\text { incompleto }\end{array}$ & 57 & $18(31,6)$ & 1,00 & & & \\
\hline Ensino Médio completo ou mais & 431 & $244(56,6)$ & $2,83(1,57 ; 5,10)$ & & & \\
\hline Tempo de atuação profissional (anos) & & & & 0,024 & & 0,031 \\
\hline $0-2$ & 37 & $14(37,8)$ & 1,00 & & 1,00 & \\
\hline 3 ou mais & 368 & $212(57,6)$ & $2,23(1,11 ; 4,48)$ & & $2,27(1,08 ; 4,80)$ & \\
\hline Caso de TB na microárea & & & & 0,006 & & \\
\hline Não & 147 & $65(44,2)$ & 1,00 & & & \\
\hline Sim & 341 & $198(58,1)$ & $1,75(1,18 ; 2,58)$ & & & \\
\hline Casos de TB assistidos nos últimos & & & & 0,004 & & 0,009 \\
\hline \multicolumn{7}{|l|}{12 meses } \\
\hline Nenhum & 258 & $124(48,1)$ & 1,00 & & 1,00 & \\
\hline 1 ou mais & 219 & $134(61,2)$ & $1,70(1,18 ; 2,46)$ & & $1,72(1,15 ; 2,59)$ & \\
\hline Realizou TDO & & & & 0,003 & & \\
\hline Não & 225 & $104(46,2)$ & 1,00 & & & \\
\hline Sim & 256 & $154(60,2)$ & $1,76(1,22 ; 2,52)$ & & & \\
\hline Preencheu ficha de acompanhamento & & & & 0,006 & & \\
\hline Não & 278 & $134(48,2)$ & 1,00 & & & \\
\hline Sim & 210 & $128(61,0)$ & $1,68(1,17 ; 2,41)$ & & & \\
\hline Capacitação sobre TB & & & & 0,004 & & \\
\hline Não & 146 & $64(43,8)$ & 1,00 & & & \\
\hline Sim & 340 & $198(58,2)$ & $1,79(1,21 ; 2,64)$ & & & \\
\hline
\end{tabular}

IC95\%: intervalo de 95\% de confiança; RC: razão de chances; TB: tuberculose; TDO: tratamento diretamente observado.

* Apenas as variáveis que apresentaram associação estatisticamente significativa com o nível de conhecimento acima da mediana nas análises univariada ou multivariada encontram-se apresentadas nesta Tabela;

** A soma em cada variável não totaliza $N=489$, devido à exclusão dos não respondentes e dos que demonstraram uma característica não aplicável ao modelo utilizado para análise.

\section{Discussão}

Constatou-se escassez de literatura científica que aborde o tema conhecimento de ACS sobre TB. Entretanto, estudo correlato a este, realizado por Maciel et al. 13, avaliou mediante questionário semiestruturado 105 ACS no Município de Vitória. O objetivo foi analisar o conhecimento deles sobre TB e a autopercepção a respeito da importância profissional no controle da doença. A conclusão apontou associação entre conhecimento mais elevado dos ACS sobre TB com tempo de atuação profissional maior ou igual a quatro anos. Comportamento semelhante ao descrito anteriormente foi observado no presente estudo. Para mais, os ACS com conhecimento situado acima da mediana de tempo de serviço, no estudo de Maciel et al. 13, demonstraram executar mais ações informativas aos pacientes com TB. Wu \& Chou 20 compararam as respostas de ACS e potenciais trabalhadores do DOTS em treinamentos realizados em Taiwan, com o objetivo de investigar o conhecimento e o estigma deles em relação à TB. Os resultados também demonstraram conhecimento mais elevado dos ACS quanto aos aspectos relacionados à sintomatologia da 
doença. Ambos os estudos apresentaram métodos que divergem da proposta do presente artigo, mas permitiram a verificação de algumas semelhanças entre a população estudada.

No tocante à frequência de acertos dos ACS a cada um dos itens do questionário, verificou-se menor quantidade no domínio TDO. A pergunta com menor frequência de acerto indica que a maioria dos ACS acredita que o TDO pode ser realizado pelos familiares, quando o correto é que a prática seja realizada exclusivamente por profissionais de saúde, para fins de notificação. O menor conhecimento a respeito do TDO indica uma lacuna na capacitação e orientação dos ACS do município e pode, quando associado a outros fatores intrínsecos ao paciente e ao serviço de saúde, proporcionar desfecho desfavorável na taxa de abandono da doença. Por sua vez, a busca ativa de pacientes faltosos e daqueles que abandonaram o tratamento como atribuição do ACS foi a única questão que apresentou $100 \%$ de acerto. Mais especificamente em relação ao domínio medidas de controle, verificou-se uma frequência insatisfatória de acertos no item sobre orientação a pacientes preferencialmente em ambientes fechados, o que representa um alerta de biossegurança. Estudo realizado em um município do Estado do Espírito Santo ${ }^{21}$ demonstra que os ACS que estiveram em contato com pacientes com TB apresentaram risco relativo 3,1 vezes superior de infecção por Mycobacterium tuberculosis, quando comparados com aqueles que não tiveram contato com casos de TB. O método utilizado para a análise dos profissionais selecionados para a amostra foi a prova tuberculínica 21 . Além disso, segundo um estudo realizado no mesmo município, que utilizou método semelhante, a infecção por M. tuberculosis entre ACS foi significativamente maior que a de seus familiares sem a mesma ocupação ${ }^{22}$. Revisão publicada por Joshi et al. 23 demonstrou prevalência de infecção latente por TB variando entre $33 \%$ e $79 \%$ do total de profissionais de todas as categorias da área da saúde. Medidas úteis para a diminuição desse risco ocupacional envolvem intervenções administrativas, adequação de infraestrutura e iniciativas que incentivem a precaução individual 24 .

Hoa et al. 25 e Minnery et al. 26, mediante a aplicação de questionários, compararam o conhecimento sobre TB entre diferentes categorias profissionais da área da saúde, dentre elas os ACS, respectivamente no Vietnã e em Lima, Peru. Ambos encontraram médias de acerto para os ACS de 13,4 em 23 questões e 9,5 ( $\pm 1,8)$ em 15 itens, respectivamente. As médias verificadas pelos pesquisadores estão abaixo das encontradas no presente estudo, tanto para o domínio TB quanto para o teste completo. No entanto, avaliações do nível de dificuldade das questões e outros testes de validação devem ser executados para melhor comparação entre os resultados.

O perfil sociodemográfico dos ACS do Município de Belo Horizonte está em conformidade com outros trabalhos que avaliaram essa categoria profissional. A maioria da população é feminina, concordando com outros estudos realizados com profissionais de saúde e ACS de municípios brasileiros 27,28,29. A idade média, escolaridade e tempo de atuação dos ACS deste estudo foram semelhantes àqueles encontrados em pesquisas realizadas em Jequié (Bahia) e em municípios dos estados do Piauí e São Paulo 28,30,31. Tal perfil que concorda com outros levantamentos demográficos realizados em municípios de todo o país, demonstra que os ACS apresentam, majoritariamente, nível de escolaridade superior ao exigido para o cargo segundo a legislação vigente 32 . O maior grau de escolaridade pode estar associado a um incremento na capacidade do ACS incorporar novas informações e transmitir o conhecimento para as famílias adscritas à sua microárea 22 . Em relação ao tempo de atuação como ACS, verifica-se concordância com o período de implantação do Programa de Agentes Comunitários de Saúde (PACS) e do Programa Saúde da Família (PSF) no município, demonstrando que $24,5 \%$ dos ACS entrevistados estão no ofício desde o início da consolidação destes programas 33 .

$\mathrm{Na}$ distribuição populacional de Belo Horizonte segundo cada IVS, verifica-se $33,7 \%$ da população residente em setor censitário de risco baixo, 39,9\% em risco médio, $19,1 \%$ em risco elevado e 7,3\% em risco muito elevado 18 . Constatou-se que a população de ACS que atua nas áreas e microáreas das diferentes categorias de IVS utilizadas no município difere da densidade populacional em cada um destes setores censitários. Como a amostra representa estatisticamente a população de ACS do município, esses dados demonstram que, proporcionalmente, a relação ACS por habitante foi maior nas áreas de risco elevado e muito elevado. Essa situação respalda o fato da implementação da ESF em Belo Horizonte ter ocorrido prioritariamente em áreas de maior vulnerabilidade em saúde 33 .

Para a operacionalização da ESF, a legislação vigente estabelece o limite máximo de 150 famílias ou 750 pessoas sob a responsabilidade de cada ACS 32,34. Belo Horizonte apresentou média de famílias acompanhadas por ACS superior ao preconizado pela legislação e aquelas observadas em outros municípios brasileiros 35,36. Esse desequilíbrio na proporção de acompanhamento de famílias por ACS pode se manifestar como fator limitante de desempenho para a realização 
das tarefas mínimas exigidas para o cargo 37 . No entanto, quando verificada a associação entre conhecimento e número de famílias acompanhadas, constatou-se maior conhecimento entre aqueles indivíduos que acompanhavam um maior número de famílias, mas não houve significância estatística.

Em contraste com a situação vigente no país quanto à dificuldade de fixação de médicos na Atenção Primária à Saúde (APS), a maior parte dos entrevistados que relatou ausências na equipe de Saúde da Família (eSF) apontou o ACS como categoria profissional mais ausente. Esse fato pode estar associado às condições geradoras de sofrimento ou estresse, relatadas pelos ACS em alguns estudos qualitativos que indicaram insatisfações relacionadas às condições de trabalho, salário e carreira, deficiências nos serviços de saúde, desconhecimento das funções, falta de reconhecimento e exposição à violência. Verifica-se que a infraestrutura associada aos serviços de saúde, a ausência de Plano de Cargos e Salários para os ACS, a falta de conhecimento a respeito da prática do tratamento supervisionado, além de outros aspectos relacionados aos pacientes podem, em conjunto, dificultar a prática do TDO 38,39.

Em relação à supervisão das ações dos ACS no controle da TB, os enfermeiros são os profissionais designados para o desempenho da função e, portanto, são citados com maior frequência pelos respondentes 17. Contudo, uma parte dos respondentes indica que outras categorias profissionais têm contribuído para o desempenho dessa função no Município de Belo Horizonte.

A quantidade de respondentes que relatou preenchimento da ficha de acompanhamento de pacientes foi menor do que aquela que afirmou ter realizado o TDO, ter assistido casos nos últimos 12 meses e ter prática no acompanhamento de pacientes dentro da microárea de atuação. Essa análise indica que, em alguns casos, pode ter ocorrido subnotificação do TDO ou execução inadequada. Levantamento realizado por Braga 40 indicou que aproximadamente $25 \%$ dos municípios brasileiros apresentavam deficiências em registrar adequadamente os dados de notificação e acompanhamento de casos de TB. O perfil traçado pelo estudo demonstrou que os estados das regiões Norte, Nordeste e Sudeste, onde há maior quantidade de casos, estão entre os mais críticos do país.

Encontra-se como limitação o fato do estudo ter sido conduzido utilizando-se um questionário não validado para avaliar o conhecimento sobre TB. Para adequação, métodos de análise estatística como a Teoria da Resposta ao Item (TRI) podem ser utilizados com o intuito de vali- dação e efeito de comparabilidade dos dados 41 . Ademais, o instrumento utilizado para medida de resultado solicitou informações a respeito de condutas profissionais, portanto, pode ter ocorrido viés de memória ou dicotomia nas respostas entre o que é realizado rotineiramente na prática laboral e o que é considerado socialmente aceitável. Outra limitação é a não observação direta do trabalho de campo, que impede a verificação da veracidade das respostas.

Foi considerada nessa pesquisa uma amostra representativa dos ACS do Município de Belo Horizonte, ponderada pela sua distribuição por DS e categorias de abono de fixação. Foi possível observar que a experiência adquirida pelo ACS no acompanhamento de casos de TB, assim como outras variáveis associadas a esta prática como a realização de TDO e o preenchimento da ficha de acompanhamento, demonstraram associação com o maior conhecimento sobre a doença, suas medidas de controle e TDO. Além disso, foi possível observar maior conhecimento associado àqueles profissionais que haviam participado de, ao menos, uma capacitação específica sobre TB, evidenciando uma vez mais a importância da educação continuada para os profissionais de saúde.

A execução desta pesquisa atende recomendações realizadas em plano estratégico, publicado pela OMS, que incentiva a produção de estudos que objetivam o aperfeiçoamento da estratégia DOTS, uma vez que o controle da TB permanece como um desafio atual devido ao alcance das metas estipuladas para atingir a sua eliminação 2 .

O conhecimento satisfatório sobre as atribuições do ACS no controle da TB demonstra que estes profissionais dominam quais devem ser suas ações no manejo dos casos de TB. Contudo, o nível de conhecimento dos ACS sobre TB, medidas de controle e TDO apresentou lacunas com relação à forma de transmissão da doença, ao público-alvo do TDO e à técnica adequada para supervisão do tratamento. Os ACS configuram-se como atores fundamentais ao serem apontados como primordiais para o controle da TB, portanto outros aspectos relacionados às facilidades, dificuldades e estigmas associados à vigilância proposta pelo TDO devem ser avaliados para melhor elucidação dessa prática, com o objetivo de adequar a formação de mão-de-obra e propor diferentes métodos didáticos para o alcance dessa finalidade. Preocupa ainda, o baixo conhecimento mensurado em relação à transmissibilidade da doença e biossegurança, considerando que a TB é um dos principais contaminantes biológicos a que estão expostos os profissionais da área de saúde. 
A perspectiva para alcance das metas da OMS é a ampliação de conhecimento para a reorientação dos profissionais, dos serviços de saúde e a gestão clínica em relação aos casos de TB. Diante disso, reforça-se a necessidade de investimento em supervisão e capacitação para aperfeiçoa-

\section{Resumen}

En este estudio transversal se evaluó el nivel de conocimiento de los agentes comunitarios de salud (ACS) sobre la tuberculosis, las medidas de control y el tratamiento directamente observado (TDO) en la ciudad de Belo Horizonte, Minas Gerais, Brasil, llevado a cabo mediante la aplicación del cuestionario en una muestra aleatoria y representativa de 489 ACS. La proporción media de ítems correctos era 74,6\%. El porcentaje promedio de respuestas correctas en el campo de la tuberculosis fue de un $81 \%$, en las medidas de control de $84,1 \%$ y 59,4\% en TDO. Había un vacío en el conocimiento acerca de la identificación de los pacientes con tuberculosis pulmonar, el público objetivo de la TDO, y la técnica adecuada para la supervisión del tratamiento. Se observó asociación entre un mayor conocimiento y experiencia de trabajo igual o superior a tres años $(R C=2,3)$ y el seguimiento de los casos en los 12 meses anteriores a la encuesta $(R C=1,7)$. Este estudio presenta información sobre el nivel de conocimiento de los ACS sobre el TDO y puede apoyar las estrategias de mejoras en las actividades de control.

Agentes Comunitarios de Salud; Conocimientos, Actitudes y Práctica en Salud; Tuberculosis mento das atividades de controle da TB. Recomenda-se, ainda, a realização de novos estudos que demonstrem o conhecimento de profissionais a respeito da doença, para efeito de comparabilidade dos dados.

\section{Colaboradores}

G. S. S. Rocha, M. G. Lima e M. R. Silveira contribuíram com a concepção do estudo, elaboração do artigo, aprovação da versão final, análise e interpretação dos dados. J. L. Moreira, K. C. Ribeiro, M. G. B. Ceccato e W. S. Carvalho contribuíram com a concepção do estudo, elaboração do artigo e aprovação da versão final.

\section{Agradecimentos}

Ao Programa Institucional de Auxílio à Pesquisa de Doutores Recém-contratados da Pró-Reitoria de Pesquisa da Universidade Federal de Minas Gerais e ao Conselho Nacional de Desenvolvimento Científico e Tecnológico (CNPq; processo 473848/2013-9) pelo auxílio financeiro. 


\section{Referências}

1. World Health Organization. Global tuberculosis report 2013. http://apps.who.int/iris/bitstream/ 10665/91355/1/9789241564656_eng.pdf (acessado em 15/Jul/2015).

2. World Health Organization. Stop TB partnership. The global plan to stop TB 2011-2015. http:// www. stoptb.org/assets/documents/global/plan/TB_ GlobalPlanToStopTB2011-2015.pdf (acessado em 15/Jul/2014).

3. Secretaria de Vigilância em Saúde, Ministério da Saúde. O controle da tuberculose no Brasil: avanços, inovações e desafios. Boletim Epidemiológico 2014; 44:1-13.

4. Hijjar MA, Gerhardt G, Teixeira GM, Procópio MJ. Retrospecto do controle da tuberculose no Brasil. Rev Saúde Pública 2007; 41:50-8.

5. Sociedade Brasileira de Pneumologia e Tisiologia. III diretrizes para tuberculose da Sociedade Brasileira de Pneumologia e Tisiologia. J Bras Pneumol 2009; 35:1018-48.

6. World Health Organization. The stop TB strategy: building on and enhancing the DOTS to meet the TB-related Millenium Development Goals. http:// www.who.int/tb/publications/2006/stop_tb_stra tegy.pdf (acessado em 26/Jun/2014).

7. Departamento de Vigilância Epidemiológica, Secretaria de Vigilância em Saúde, Ministério da Saúde. Manual de recomendações para o controle da tuberculose no Brasil. Brasília: Editora MS; 2011.

8. Departamento de Atenção Básica, Secretaria de Atenção à Saúde, Ministério da Saúde. Vigilância em saúde: dengue, esquistossomose, hanseníase, malária, tracoma e tuberculose. Brasília: Editora MS; 2008. (Cadernos de Atenção Básica).

9. Pereira IC, Oliveira MAC. O trabalho do agente comunitário na promoção da saúde: revisão integrativa da literatura. Rev Bras Enferm 2013; 66:412-9.

10. Machado NJ. Epistemologia e didática. 7ạ Ed. São Paulo: Cortez Editora; 2011.

11. Woith WM, Volchenkov G, Larson JL. Russian healthcare workers' knowledge of tuberculosis and infection control. Int J Tuberc Lung Dis 2010; 14:1489-92.

12. Kiefer E, Shao T, Carasquillo O, Nabeta P, Seas C. Knowledge and attitudes of tuberculosis management in San Juan de Lurigancho district of Lima, Peru. J Infect Dev Ctries 2009; 10:783-8.

13. Maciel ELN, Vieira RCA, Milani EC, Brasil M, Fregona G, Dietze R. O agente comunitário de saúde no controle da tuberculose: conhecimento e percepções. Cad Saúde Pública 2008; 24:1377-86.

14. Prefeitura de Belo Horizonte. Lei no 7.238, de 30 de dezembro de 1996. Dispõe sobre o quadro especial da Secretaria Municipal de Belo Horizonte, institui o plano de carreira dos servidores da saúde da Prefeitura Municipal de Belo Horizonte, estabelece a respectiva tabela de vencimentos e dá outras providências. Diário Oficial do Município 1996; 31 out.

15. Prefeitura de Belo Horizonte. Decreto no 12.924, de 1 o de novembro de 2007. Regulamenta o art. 12 da Lei no 9.443, de 18 de outubro de 2007. Diário Oficial do Município 2007; 1 nov.
16. Prefeitura de Belo Horizonte. Lei no 10.252, de 13 de setembro de 2011. Concede reajustes remuneratórios aos servidores e empregados públicos da administração direta e indireta do poder executivo e dá outras providências. Diário Oficial do Município 2011; 13 set.

17. Departamento de Vigilância Epidemiológica, Secretaria de Vigilância em Saúde, Ministério da Saúde. Tratamento Diretamente Observado (TDO) da tuberculose na atenção básica: protocolo de enfermagem. Brasília: Editora MS; 2011.

18. Secretaria Municipal de Saúde de Belo Horizonte. Tuberculose: orientações para agentes comunitários de saúde. Belo Horizonte: Secretaria Municipal de Saúde de Belo Horizonte; 2012.

19. Secretaria Municipal de Saúde de Belo Horizonte. Índice de vulnerabilidade da saúde 2012. Belo Horizonte: Secretaria Municipal de Saúde de Belo Horizonte; 2013.

20. Wu P, Chou P. Characteristics of directly observed treatment short-course workers in Taiwan. J Chin Med Assoc 2013; 76:48-52.

21. Moreira TR, Zandonade E, Maciel ELN. Risco de infecção tuberculosa em agentes comunitários de saúde. Rev Saúde Pública 2010; 44:332-8.

22. Rodrigues PM, Moreira TR, Moraes AKL, Vieira RCA, Dietze R, Lima RCD, et al. Infecção por Mycobacterium tuberculosis entre agentes comunitários de saúde que atuam no controle da TB. J Bras Pneumol 2009; 35:351-8.

23. Joshi R, Reingold AL, Menzies D, Pai M. Tuberculosis among Health-care workers in low- and middle-income countries: a systematic review. PLoS Med 2006; 3:e494.

24. Fica A, Cifuentes M, Ajenjo MC, Jemenao MI, Zambrano A, Febré N, et al. Tuberculosis en el personal de salud. Rev Chil Infect 2008; 25:243-55.

25. Hoa NP, Diwan VK, Thorson AE. Diagnosis and treatment of pulmonary tuberculosis at basic health care facilities in rural Vietnam: a survey of knowledge and reported practices among health staff. Health Policy 2005; 72:1-8.

26. Minnery M, Contreras C, Pérez R, Solórzano N, Tintaya K, Jimenez J, et al. A cross sectional study of knowledge and attitudes towards tuberculosis amongst front-line tuberculosis personnel in high burden areas of Lima, Peru. PLoS One 2013; 8:e75698.

27. Ferraz L, Aerts DRGC. O cotidiano de trabalho do agente comunitário de saúde no PSF em Porto Alegre. Ciênc Saúde Coletiva 2005; 10:347-55.

28. Resende MC, Azevedo EGS, Lourenço LR, Faria LS, Alves NF, Farina NP, et al. Saúde mental e ansiedade em agentes comunitários que atuam em saúde da família em Uberlândia (MG, Brasil). Ciênc Saúde Coletiva 2011; 16:2115-22.

29. Crispim JA, Scatolin BE, Silva LMC, Pinto IC, Palha PF, Arcêncio RA. Agente comunitário de saúde no controle da tuberculose na Atenção Primária à Saúde. Acta Paul Enferm 2012; 25:721-7. 
30. Moura MS, Carvalho CJ, Amorim JTC, Marques MFSS, Moura LFAD, Mendes RF. Perfil e práticas de saúde bucal do agente comunitário de saúde em municípios piauienses de pequeno porte. Ciênc Saúde Coletiva 2010; 15:1487-95.

31. Mascarenhas CHM, Prado FO, Fernandes MH. Fatores associados à qualidade de vida de agentes comunitários de saúde. Ciênc Saúde Coletiva 2013; 18:1375-86.

32. Casa Civil, Presidência da República. Lei no 11.350, de 5 de outubro de 2006. Regulamenta o parágrafo 5o do art. 198 da Constituição, dispõe sobre o aproveitamento de pessoal amparado pelo parágrafo único do art. 2o da Emenda Constitucional no 51, de 14 de fevereiro de 2006, e dá outras providências. Diário Oficial da União 2006; 5 out.

33. Turci MA, organizadora. Avanços e desafios na organização da atenção de saúde em Belo Horizonte. Belo Horizonte: Secretaria Municipal de Saúde de Belo Horizonte; 2008.

34. Ministério da Saúde. Portaria no 1.886/GM, de 18 de dezembro de 1997. Aprova as normas e diretrizes do Programa de Agentes Comunitários de Saúde e do Programa de Saúde da Família. Diário Oficial da União 1997; $18 \mathrm{dez}$.

35. Gomes KO, Cotta RMM, Cherchiglia ML, Mitre SM, Batista RS. A práxis do Agente Comunitário de Saúde no contexto do Programa Saúde da Família: reflexões estratégicas. Saúde Soc 2009; 18:744-55.
36. Santos TMMG, Nogueira LT, Arcêncio RA. Atuação de profissionais da Estratégia Saúde da Família no controle da tuberculose. Acta Paul Enferm 2012; 25:954-61.

37. Martines WRV, Chaves EC. Vulnerabilidade e sofrimento no trabalho do Agente Comunitário de Saúde no Programa de Saúde da Família. Rev Esc Enferm USP 2007; 41:426-33.

38. Wai MFP, Carvalho AMP. O trabalho do Agente Comunitário de Saúde: fatores de sobrecarga e estratégias de enfrentamento. Rev Enferm UERJ 2009; 17:563-8.

39. Lopes DMQ, Beck CLC, Prestes FC, Weiller TH, Colomé JS, Silva GM. Agentes Comunitários de Saúde e as vivências de prazer - sofrimento no trabalho: estudo qualitativo. Rev Esc Enferm USP 2012; 46:633-40.

40. Braga JU. Vigilância epidemiológica e o sistema de informação da tuberculose no Brasil, 2001-2003. Rev Saúde Pública 2007; 41:77-88.

41. Andrade DF, Tavares HR, Valle RC. Teoria de resposta ao item: conceitos e aplicações. São Paulo: Associação Brasileira de Estatística; 2000.

Recebido em 25/Jul/2014

Versão final reapresentada em 12/Nov/2014

Aprovado em 05/Fev/2015 